\title{
El Aprovechamiento de la Fauna como Instrumental Óseo en Punta Entrada y Parque Nacional Monte León (Provincia de Santa Cruz, Argentina)
}

\author{
Faunal Use for Bone Tools. Punta Entrada and Monte León National Park Collections \\ (Santa Cruz province, Argentina).
}

Natacha Buci e Isabel Cruz ${ }^{\text {ii }}$

\section{RESUMEN}

Este trabajo representa una primera aproximación al aprovechamiento de la fauna en la producción de instrumentos óseos en las localidades de Punta Entrada y Parque Nacional Monte León, ubicadas en el litoral Atlántico, al sur del río Santa Cruz. El material está asociado a ocupaciones de los últimos 2000 años, incluyendo una señal temprana de ca. 5700-4000 AP en Monte León. Se clasifica la muestra en grupos morfológicos y en cada uno de ellos, a su vez, se determina el hueso utilizado como soporte artefactual. En el caso del grupo más representativo, que es el de los retocadores, también se establece la estructura métrica. En ambas localidades se observan evidencias de una tecnología ósea desarrollada. La homogeneidad evidente en la selección de la materia prima, está asociada a una gran variabilidad morfológica que sugiere la orientación de los instrumentos óseos hacia diferentes actividades del modo de vida de los cazadores-recolectores locales.

Palabras Clave: Fauna, Tecnología ósea, Hueso-soporte.

\section{ABSTRACT}

This paper is a first approach to faunal use in the production of bone tools in Punta Entrada and Parque Nacional Monte León locations, placed in the Atlantic coast, south of Santa Cruz River.The assemblage is associated to human occupations which took place in the last 2000 years, including an early signal ca. 5700-4000 BP in Monte León. The sample is classified in morphofunctional groups and we identified the bone used as raw material for each case. In the most representative group, that of pressure flakers, metrical structure is also determined. Evidences of well developed bone technology can be seen in both localities. Homogeneity shown in raw material selection is linked to a wide morphological variety which suggests bone tool were related with different activities of local hunter-gatherers.

Key Words: Fauna, Bone tools, Bone raw material.

i CONICET-INAPL. 3 de febrero 1378, (1426) Buenos Aires, Argentina. Correo-e: natachabuc@gmail.com

ii UARG-UNPA- Lisandro de la Torre 1070, (9400) Río Gallegos, Santa Cruz, Argentina. Correo-e: isabelzooarqueologia@ gmail.com

Recibido: 14-11-2012 Revisado: 07-05-2013 Aceptado: 08-11-2013 


\section{INTRODUCCIÓN}

La explotación de los recursos faunísticos es uno de los factores que condicionan la organización espacial y tecnológica de los cazadores-recolectores. En este trabajo nos proponemos abordar un aspecto del aprovechamiento de la fauna: la utilización de sus huesos como materia prima, presentando los instrumentos óseos de Punta Entrada (PE) y Parque Nacional Monte León (PNML), dos localidades ubicadas sobre el litoral atlántico de Argentina al sur del río Santa Cruz. PE es una forma de acreción marina enmarcada por un acantilado inactivo que, como gran parte de la costa sur del estuario, está disectado por amplios cañadones originados por la acción fluvial. En este sector predominan los médanos activos e inactivos y la vegetación es una estepa arbustiva baja. El paisaje costero del PNML se encuentra profundamente afectado por una intensa acción erosiva, marina y fluvial. En la zona predominan las estepas arbustivas, especialmente los matorrales de mata negra (Junellia tridens).Ambas localidades poseen un registro arqueológico conformado por depósitos a cielo abierto tanto en superficie como en estratigrafía, con cronologías que abarcan los últimos 2000 años AP y que incluyen una señal temprana de ca.5700-4000 AP en PNML (Cruz et al. 2012, Muñoz et al. 2009).

Los depósitos contienen altos porcentajes de restos de pinnípedos, que pueden alcanzar hasta el $90 \%$ de la representación taxonómica (Cruz et al. 20I2, Muñoz et al. 20I2). La fauna terrestre, en cambio, está poco representada, y los restos de guanaco (Lama guanicoe) constituyen menos del I\% en PE y entre el 8-9\% en PNML. Los huesos de aves marinas $y$ terrestres son escasos y en muchos casos corresponden a intrusiones actuales en los depósitos arqueológicos. Sin embargo, el análisis isotópico de restos humanos de la zona muestra una dieta con consumo predominante de recursos terrestres y una ingesta baja de alimentos marinos (Suby et al. 2009).

En este trabajo, nuestro objetivo es realizar una primera caracterización del instrumental óseo recuperado en ambas localidades, como una vía más para discutir diversos aspectos de la relación de los cazadores-recolectores con el ambiente y la fauna en este sector de la costa patagónica. Para ello, presentamos los grupos morfológicos registrados, la selección de huesos-soporte y los primeros resultados del análisis microscópico.

\section{METODOLOGÍA}

La muestra analizada está formada por 2 I piezas de PE y 3 de PNML (Tabla I). Los grupos morfológicos se caracterizaron de acuerdo a la bibliografía disponible (Bird 1993, Scheinsohn 1997, 2010a). La determinación anatómica y taxonómica del hueso soporte se realizó con la colección comparativa de la Unidad Académica Río Gallegos de la Universidad Nacional de la Patagonia Austral. Para el análisis microscópico se utilizó lupa binocular a 20X (Instituto Nacional de Antropología y Pensamiento Latinoamericano) y microscopio metalográfico a 50X, 100X y 200X (Comisión Nacional de Energía Atómica).

\begin{tabular}{|c|c|c|c|}
\hline Código & Hueso-Soporte & Taxón & $\begin{array}{l}\text { Grupo- } \\
\text { morfológico }\end{array}$ \\
\hline PE I & hueso & Cetáceo & Cuña \\
\hline PE 2 & hueso & Cetáceo & Afilador \\
\hline PE 3 & costilla & $\begin{array}{c}\text { Mammalia } \\
\text { terrestre }\end{array}$ & Cuña \\
\hline PE 4 & tibiatarso proximal & Rhea americana & Marcado perimetral \\
\hline PE 5 & $\begin{array}{c}\text { tarso-metatarso } \\
\text { distal }\end{array}$ & Rhea americana & $\begin{array}{l}\text { Aserrado } \\
\text { perimetral }\end{array}$ \\
\hline PE 6 & asta & $\begin{array}{l}\text { Hippocamelus } \\
\text { bisulcus }\end{array}$ & $\begin{array}{l}\text { Aserrado } \\
\text { perimetral }\end{array}$ \\
\hline PE 7 & ulna proximal & Lama guanicoe & Retocador \\
\hline PE 8 & metapodio proximal & Lama guanicoe & Retocador \\
\hline PE 9 & metapodio distal & Lama guanicoe & Retocador \\
\hline PE 10 & metacarpo proximal & Lama guanicoe & Retocador \\
\hline PE II & metatarso proximal & Lama guanicoe & Retocador \\
\hline PE 12 & diáfisis & Mammalia & Punta Roma \\
\hline PE I 3 & hueso & Mammalia & punta arpón \\
\hline PE I4 & hueso & Mammalia & punta arpón \\
\hline PE I5 & hueso & Mammalia & punta roma \\
\hline PE 16 & hueso & Mammalia & Indet \\
\hline PE 17 & diáfisis & Lama guanicoe & $\begin{array}{c}\text { Punta cóncavo- } \\
\text { convexa }\end{array}$ \\
\hline PE 18 & hueso & Mammalia & Punta roma \\
\hline PE 19 & tibia-tarso proximal & Rhea americana & Marcado perimetral \\
\hline PE 20 & hueso & Mammalia & $\begin{array}{c}\text { Punta cóncavo- } \\
\text { convexa }\end{array}$ \\
\hline PE 21 & hueso & Mammalia & Gancho propulsor \\
\hline PE 22 & metapodio & Mammalia & Punta roma \\
\hline PML I & $\begin{array}{l}\text { metapodio } \\
\text { pxroximal }\end{array}$ & Lama guanicoe & Retocador \\
\hline PML 2 & radio ulna o húmero & Lama & Retocador \\
\hline
\end{tabular}

Tabla 1: Muestra de Punta Entrada (PE) y Parque Nacional Monte León (PML). Determinación morfológica y física.

Table 1: Punta Entrada (PE) y Parque Nacional Monte León (PML) Samples. Morphologic and physical determination. 


\section{RESULTADOS}

Todos los instrumentos óseos fueron recuperados en superficie, en asociación a contextos arqueológicos. Su estado de conservación, medido a través de la meteorización (Behrensmeyer 1978), es acorde con una exposición prolongada: el $92 \%$ de las piezas muestra signos de meteorización. Sin embargo, siete de las piezas de PE y dos de PNML presentan marcas de raíces, lo cual también ha sido registrado en los conjuntos arqueofaunísticos e interpretado como evidencia de que los depósitos estuvieron cubiertos por sedimentos durante al menos parte de su historia (Cruz et al. 20I2).

Por su dominancia numérica, entre los grupos morfológicos se destacan las puntas romas denominadas retocadores (Bird 1993, Borella y Buc 2009, Scheinsohn 2010a), varios de los cuales presentan incisiones sobre las caras laterales, lo cual constituye un rasgo común en Patagonia (Bird 1993). También se recuperaron arpones monodentados (cf. Scheinsohn 2010b), cuñas -una de ellas también definida como calibrador, afilador o pulidor por sus marcas en la cara cóncava (cf. Outes 1918)-, puntas cóncavo-convexas, y un gancho de propulsor (cf. Scheinsohn 20I0a) (Figura I).

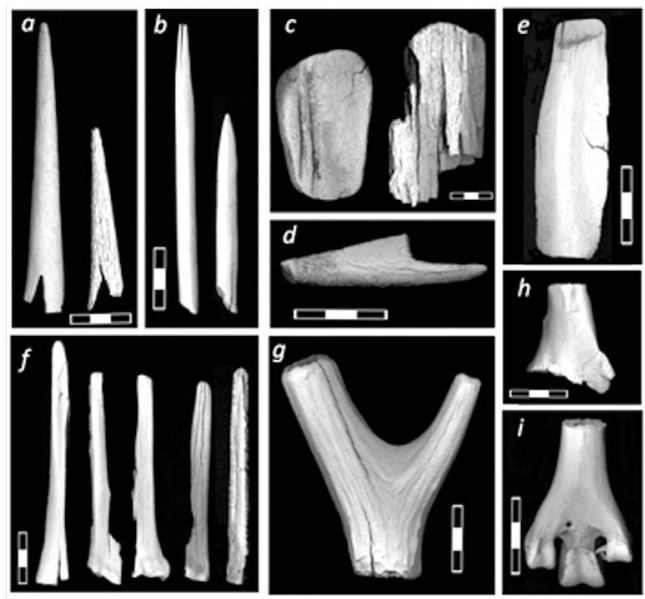

Figura 1: Grupos morfológicos. a) arpones, b) puntas cóncavoconvexas, c) cuñas en cetáceo, con y sin surcos, d) gancho de propulsor, e) cuña en costilla, $\mathrm{f}$ ) retocadores, $\mathrm{g}$ ) asta con aserrado perimetral, h) tibia-tarso con marcado perimetral, i) tarsometatarso con aserrado perimetral.

Figure 1: Morphological groups. a) harpoons, b) concave-convex points, c) wedges made on cetacean bone, with and without groove, d) hook of spearthrower, e) wedge made on rib, f) pressure flakers, g) antler sawed in the perimeter, h) tibia-tarsal bone marked in the perimeter, i) tarsal-metatarsal sawed in the perimeter.
Cada grupo morfológico presenta fuertes recurrencias en la estructura física. Los retocadores (Figura If) se realizaron principalmente en metapodios de guanaco y su formatización buscó mantener la geometría original del hueso: la estructura métrica muestra poca variabilidad en el ancho máximo y espesor del ápice (Figura 2) (ver una situación similar en Borella y Buc 2009, y Marani y Cardillo 2010). Teniendo en cuenta el trabajo de Scheinsohn (1997), podemos decir que se eligieron las características estructurales de este elemento que lo hacen adecuado para actividades de presión, como las involucradas en la regularización de filos líticos. Los arpones (Figura la) se confeccionaron con huesos de mamíferos marinos, posiblemente cetáceos, tal como ha sido registrado en otros contextos, aprovechando su elasticidad como materia prima (Borrero y Borella 2010, Moreno 2008, Scheinsohn 1997, 2010b). Este mismo hueso fue utilizado en la elaboración de dos cuñas (Figura Ic). En el caso de aquella pieza con surcos, la costilla de cetáceo en estado seco, por la cantidad de tejido esponjoso, se vuelve un material abrasivo que se ajusta a la idea de que haya sido utilizada para "afilar" puntas $\circ$ astiles. También se registró la presencia de asta de huemul (Figura I) (Cruz et al. 2010) que habría sido trasladada a la costa aprovechando las propiedades de este soporte como materia prima de instrumentos (Guthrie 1983).

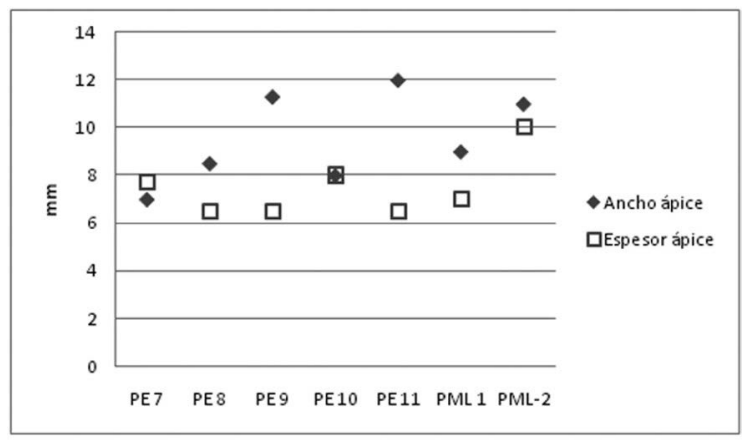

Figura 2: Estructura métrica de los retocadores. Punta Entrada (PE) y Parque Nacional Monte León (PML).

Figure 2: Metrical structure of pressure flakers. Punta Entrada (PE) and Parque Nacional Monte León (PML).

Una primera aproximación microscópica permitió determinar que, a pesar de la intensa alteración post-depositacional, las piezas conservan huellas de manufactura y/o uso, confirmando su modificación intencional. Fue posible establecer 
una clara diferencia entre las piezas con aserrado perimetral y aquellas que presentan el denominado "marcado" (cf. Acosta 2000). Entre las primeras se aprecian las huellas de corte en el perímetro, sin rastros que denoten uso, mientras que las segundas presentan superficies redondeadas, compactadas sobre el filo y con lascados sobre la cara del elemento (véase también Acosta et al. en este volumen). La muestra analizada incluye dos tibia-tarsos de ñandú con marcado perimetral (Figura Ih), que podrían explicarse tanto por la extracción de médula ósea (cf. Borrero 1994-1995) como por su utilización como instrumentos (cf. Hadjuk y Lezcano 2005; Sidéra 2010). Si bien este aspecto podría determinarse analizando el patrón microscópico de las piezas, este estudio no pudo realizarse debido a la meteorización de la superficie. El aserrado en un tarso-metatarso de ñandú (Figura li) y en el asta de huemul (Figura Ig) indicaría la producción local de instrumentos óseos, siguiendo la propuesta de Acosta (2000).

\section{DISCUSIÓNY CONCLUSIONES}

El análisis efectuado permitió un primer acercamiento a la tecnología ósea de PE y PNML. La muestra se destaca por su diversidad, que incluye grupos morfológicos típicos de ámbitos acuáticos, como los arpones (Moreno 2008, Scheinsohn $2010 b)$ y de estepa, como el gancho de propulsor asociado a la captura abierta terrestre (Catellain 1997, Scheinsohn 2010a) y las puntas cóncavoconvexas largas que estarían vinculadas a un sistema de armas tipo lanza de mano o con propulsor (cf. Ratto 2003, Rozoy 1992). Si bien hay pocos ítems por caso, notamos cierta homogeneidad al interior de cada uno de ellos en cuanto a estructura físicomorfológica y métrica. Se destaca el predominio del guanaco como soporte óseo, seguido por los huesos de cetáceo, conformando conjuntos taxonómicos diferentes a los arqueofaunísticos mencionados.

En síntesis, los resultados de este trabajo sugieren que para los últimos 2000 años existía en ambas localidades una tecnología ósea que incluye la selección de materia prima ósea para la producción local de instrumentos y una variabilidad morfológica que los vincula a otras tecnologías y a la explotación de recursos realizada por los cazadores-recolectores locales. Todo esto concuerda, en principio, con un momento en el que la explotación de la materia prima ósea está bien desarrollada (Scheinsohn 1997).

Agradecimientos: Al departamento de Microscopía de CNEA, especialmente a Ricardo Montero por el acceso al microscopio metalográfico, y a Edgardo Cabanillas. A los evaluadores, cuyos comentarios contribuyeron a clarificar el trabajo. Los trabajos en Punta Entrada y P. N. Monte León fueron financiados por los proyectos PIP/CONICET I I2-20080I-00996 y UNPA 29/A260.

\section{BIBLIOGRAFÍA}

Acosta, A. 2000. "Huellas de corte relacionadas con la manufactura de artefactos óseos en el nordeste de la provincia de Buenos Aires". Relaciones XXV: I59-I78.

Acosta, A., N. Buc y L. Mucciolo. 2014. "Elementos óseos con aserrado perimetral. El caso del humedal del río Paraná inferior". Revista Chilena de Antropología. En este volumen.

Bird, J. B. 1993. Viajes y Arqueología en Chile Austral. Universidad de Magallanes, Chile.

Behrensmeyer, A. K., 1978. “Taphonomic and Ecological Information from Bone Weathering”. Paleobiology 4:150-162.

Borella F. y N. Buc. 2009. "Ópticas y ópticos". En Arqueología de Patagonia: una mirada desde el último confin, editado por M. Salemme, F. Santiago, M. Alvarez, E. Piana, M.Vazquez y M.E. Mansur, pp. 42I-432. Editorial Utopías, Ushuaia.

Borrero, L. A. 1994-1995. "Arqueología de la Patagonia". Palimpsesto 4: 9-69.

Borrero, L.A. y F. Borella. 2010. "Harpoons and travellers: Fuegian ethnographic collections and the recent archaeological record". Before Farming 3: I- 14.

Cattellain, P. 1997."Hunting during the Upper Paleolithic: Bow, Spearthrower or Both?". En Projectile Technology, editado por H. Knetcht, pp. 213-240. Plenum Press, Nueva York.

Cruz, I.; A. S. Muñoz y M. S. Caracotche 2010."Un artefacto en asta de huemul (Hippocamelus bisulcus) en depósitos arqueológicos costeros. Implicaciones para la movilidad humana y la distribución de la especie". Magallania 38(I): 289-296.

Cruz, I.; A. S. Muñoz y P. A. Lobbia 20I2. "La explotación de recursos marinos en la costa de Patagonia continental: los restos de vertebrados en depósitos de Punta Entrada y Monte León (Santa Cruz,Argentina)". Revista de estudios marítimos y sociales 4 . En prensa.

Guthrie, D. 1983 “Osseous Projectile Point”. En Hunters and their Prey, editado por J. Clutton-Brock y C. Grigson, pp. 274294. BAR International Series 163, Oxbow, Oxford.

Hajduk, A. y M. J. Lezcano. 2005. "Un "nuevo" integrante del elenco de instrumentos óseos de Patagonia: los machacadores óseos". Magallania 33(I):63-80.

Marani, H. y M. Cardillo. 2010."Retocadores óseos de Saco Viejo (Río Negro, Argentina)". En Zooarqueología a Principios del Siglo XXI: Aportes Teóricos, Metodológicos y Casos de Estudio, editado por M.A. Gutiérrez, M. De Nigris, P.M. Fernández, M. 
Giardina, A.F. Gil, A. Izeta, G. Neme y H.D. Yacobaccio, pp. 453-458. Editorial del Espinillo, Buenos Aires.

Moreno, E. 2008. Arqueología y Etnohistoria de la Costa de la Patagónica Central en el Holoceno Tardío. Fondo Editorial Provincial, Chubut.

Muñoz, A.S.; M. S. Caracotche e I. Cruz 2009. "Cronología de la costa al sur del río Santa Cruz:nuevas dataciones en Punta Entrada y Parque Nacional Monte León (Provincia de Santa Cruz,Argentina)". Magallania 37(I): 39-43.

Muñoz, A. S.; I. Cruz; C. R. Lemaire y A. Pretto 2012. "Los restos arqueológicos de pinnípedos de la desembocadura del río Santa Cruz (Punta Entrada, costa atlántica de Patagonia) en perspectiva regional". Actas de las VIII Jornadas de Arqueología de la Patagonia. En prensa.

Outes, F. 1918. "Nuevo jalón septentrional en la dispersión de representaciones plásticas de la cuenca paranaense y su valor indicador". Anales de la Sociedad Científica Argentina LXXXV:53-66.

Ratto, N. 2003. Estrategias de Caza y Propiedades de Registro Arqueológico en la Puna de Chaschuil (Dpto. de Tinogasta, Catamarca, Argentina). Tesis para optar por el título de Doctor de la Universidad de Buenos Aires. Facultad de Filosofía y Letras.

Rozoy, J-C. 1992. "Expérimentation de lancer de sagaies avec le propulseur". Bulletin des Chercheurs de la Wallonie XXXII:I69-I84
Scheinsohn, V. 1997. Explotación de materias primas óseas en la Isla grande de Tierra del Fuego.Tesis para optar por el título de Doctor de la Universidad de Buenos Aires. Facultad de Filosofía y Letras.

2010a. "Down to the Bone:Tracking Prehistoric Bone Technology in Southern Patagonia". En Ancient and Modern Bone Artefacts from America to Russia, editado por A. Legrand-Pineau, I. Sidéra, N. Buc, E. David y V. Scheinsohn, Pp. I-6. BAR International Series 2136, Oxbow, Oxford.

2010b. "The Good, the Bad and the Ugly: Prehispanic Harpoon Heads from Beagle Channel, Isla Grande de Tierra del Fuego (Patagonia,Argentina)". En Ancient and Modern Bone Artefacts from America to Russia, editado por A. Legrand-Pineau, I. Sidéra, N. Buc, E. David y V. Scheinsohn, pp. 295-302. BAR International Series 2136, Oxbow, Oxford.

Sidéra, I. 2010. "Early Neolithic and Chalcolithic Crude Adzes. A Technological and Use-wear Focus on an Unknown Artefact Type from Near-East to Western Europe". En Ancient and Modern Bone Artefacts from America tu Russia, editado por A. Legrand-Pineau, I. Sidéra, N. Buc, E. David and V. Scheinsohn, Pp. 227-233. BAR International Series 2136, Oxbow, Oxford.

Suby, J., R. Guichón y A. F. Zangrando. 2009. "El registro biológico humano de la costa meridional de Santa Cruz". Revista Argentina de Antropología Biológica I I:109-124. 